\title{
Wideband analytical equivalent circuit for one-dimensional periodic stacked arrays
}

\author{
Carlos Molero, ${ }^{*}$ Raúl Rodríguez-Berral ${ }^{\dagger}$ and Francisco Mesa ${ }^{\ddagger}$ \\ Department of Applied Physics 1, University of Sevilla, 41012 Seville, Spain \\ Francisco Medina ${ }^{\S}$ \\ Department of Electronics and Electromagnetism, University of Sevilla, 41012 Seville, Spain \\ Alexander B. Yakovlev"l \\ Department of Electrical Engineering, Center for Applied Electromagnetic Systems Research (CAESR), \\ University of Mississippi, University, Mississippi 38677, USA
}

(Received 14 July 2015; published 15 January 2016)

\begin{abstract}
A wideband equivalent circuit is proposed for the accurate analysis of scattering from a set of stacked slit gratings illuminated by a plane wave with transverse magnetic or electric polarization that impinges normally or obliquely along one of the principal planes of the structure. The slit gratings are printed on dielectric slabs of arbitrary thickness, including the case of closely spaced gratings that interact by higher-order modes. А П-circuit topology is obtained for a pair of coupled arrays, with fully analytical expressions for all the circuit elements. This equivalent $\Pi$ circuit is employed as the basis to derive the equivalent circuit of finite stacks with any given number of gratings. Analytical expressions for the Brillouin diagram and the Bloch impedance are also obtained for infinite periodic stacks.
\end{abstract}

DOI: 10.1103/PhysRevE.93.013306

\section{INTRODUCTION}

The features of the scattering of electromagnetic waves by periodic structures made of dielectric or metallic materials have been studied for a long time at frequencies ranging from the optical [1-5] to the microwave [6-9] regime. The interest in these structures lies in their ability to control the polarization, reflection, transmission, and absorption of electromagnetic waves. Although fully dielectric implementations are advisable at optical frequencies [10] and feasible at microwave frequencies [11], many of the applications found in the range from microwave to terahertz frequencies involve metallic patterns printed on dielectric slabs. These patterns are periodic distributions of strips or patches or, alternatively, periodic distributions of slits or slots etched on metal films coating dielectric slabs. Structures of this kind have been commonly used as frequency-selective surfaces [12,13], polarizers $[14,15]$, or high-impedance surfaces [16,17], to mention a few relevant examples.

The interest in these periodic structures was likewise raised by the discovery of the so-called optical extraordinary transmission [18], which was first associated with the characteristics of wave propagation at optical frequencies [18,19], although soon it was found and measured at millimeter wave bands [20,21]. In parallel with the previous works, some researchers have proposed an alternative point of view based on classical concepts of microwave circuit theory [6-9,22,23]. This point of view was also implicitly adopted in some early papers on extraordinary transmission [24,25] and explicitly

\footnotetext{
*cmolero@us.es

†rrberral@us.es

†mesa@us.es

§medina@us.es

"yakovlev@olemiss.edu
}

employed in studies dealing with enhanced transmission through small diaphragms inside closed waveguides [26-28]. The possibilities of this perspective to provide a simple and accurate explanation of the extraordinary transmission behavior and other exotic effects was initially employed by some of the authors in papers that reported equivalent-circuit models to accurately obtain the transmission properties of periodic arrays of holes [29] or slits [30] made in thin or thick metal screens.

In most circuit-model-oriented papers, after the unit cell of the periodic structure is defined, the problem is reduced to the solution of a typical waveguide-discontinuity problem, which, in general, can be substituted by an equivalent-circuit network that accurately reproduces the transmission and reflection behavior of the original structure. Moreover, the corresponding network topology provides good physical insight into the involved electromagnetic phenomena and helps to predict other potentially interesting behaviors of the structure when any structural parameter is varied. Another apparent advantage of the analytical equivalent-circuit approach is the short CPU time required to achieve numerical results (usually in the range of milliseconds), in contrast to the great computational effort demanded by commercial electromagnetic full-wave simulators (ranging from several minutes to hours). In the equivalent-circuit approach, the propagating fields are modeled by transmission lines, while the reactive fields around the discontinuities are accounted for by lumped capacitance or inductance elements, which are a good approximation for electrically short discontinuity elements at the operating wavelength $[6,8]$. Many equivalent-circuit proposals found in the literature do not give analytical expressions for the lumped elements, but instead their values are usually extracted from intensive external full-wave simulations. In other instances [17,22,31-34] some analytical formulas are provided, but they have a limited range of applications that typically does not extend beyond the onset of the first grating lobe 
(diffraction regime) or are valid only in the subwavelength regime. Further extension into the diffraction regime requires more sophisticated models, usually requiring that some of the circuit parameters are frequency dependent. In this way, some proposals [35-39] account for the diffraction orders as different modes of the equivalent waveguide, whose modal impedances provide the explicit closed-form frequency dependence of such circuit parameters. A further step was given in [40], where, using a multimodal analysis, fully analytical expressions for all the circuit elements are provided (both lumped and distributed). In that work, self-consistent models for single metallic one-dimensional (1D) strip or slit arrays printed on dielectric layers were obtained by a rigorous method, and these models were extended to other periodic configurations such as T-shaped 1D periodic structures [41] and 2D arbitrarily shaped periodic structures [42]. Using a similar multimodal analysis, diffractive effects were also considered in [38] for periodic distributions of slits made in thick metal screens. Another type of multimodal circuit models was derived, for instance, in [43]. A nice example of how circuit models can predict interesting physical effects is given in [44], where the anomalous extraordinary transmission is explained as a consequence of the behavior of the circuit model associated with a 1D grating excited by transverse electric (TE)-polarized waves. It should be mentioned here, to give proper credit to alternative paths to obtain highly efficient solutions for this kind of problem, that analytical or quasianalytical solutions to the strip-like scattering problem are available not just in the form of equivalent circuits. A number of elegant and sophisticated mathematical tools based on Wiener-Hopf and Riemann-Hilbert techniques as well as a variety of regularization methods to solve singular integral equations have been proposed, leading to extremely fast and accurate computer codes (a few examples of this type of work can be found in [45]-[53]). Equivalent circuits are usually less accurate but they have the advantage of being significantly simpler than the above-mentioned approaches.

In all the above-mentioned models, the structure under analysis involved a single grating surface, leaving aside those situations where coupled gratings are of great interest [7,9,25,54-59]. The latter structures have been proposed for design of artificial materials, filters, and polarizers, and very recently they have been employed to simulate materials with a high permittivity (artificial dielectrics) over a wide terahertz frequency range $[60,61]$. Some of the authors of the present work have already dealt [40,57] with a single pair of coupled identical strip-like gratings by using an even or odd excitation analysis [62] (this allows us to reduce the original coupled-array problem to two subproblems with a single grating surface and with an electric or magnetic wall in the corresponding symmetry plane), and a strategy similar to the one followed in this paper has very recently been implemented to approximately model fishnet structures [63]. Experimental studies of this type of structure can also be found for other 1D [54] and 2D [64] configurations.

As the obtaining of analytical solutions for stacked gratings is a subject of considerable interest, the purpose of this work is first to present a systematic method to derive a wideband equivalent $\Pi$ circuit for a pair of 1D slit coupled arrays such as that shown in Fig. 1 under normal or oblique transverse

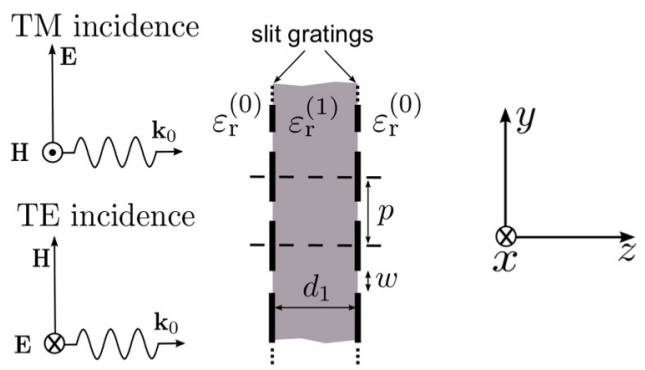

FIG. 1. Cross section of a pair of periodic coupled slit arrays under TM or TE normal incidence. The metal strips are infinitely thin and infinitely long along the $x$ direction. Parameters: period of the arrays, $p$; slit width, $w$; distance between gratings (or dielectric thickness), $d_{1}$; relative permittivity of the dielectric slab, $\varepsilon_{\mathrm{r}}^{(1)}$; and relative permittivity of the external medium, $\varepsilon_{\mathrm{r}}^{(0)}$.

magnetic (TM) or transverse electric (TE) incidence and then to use this $\Pi$ circuit as the basis to obtain the equivalent circuit for the stacked structure. Fully analytical expressions for all the circuit elements are rigorously derived following the guidelines of our previous work on this topic [40,57]. This circuit model can also be used to study infinite periodic stacks of $1 \mathrm{D}$ gratings. The dispersion relation of a Bloch mode and the Bloch impedance of the structure is easily calculated from the unit-cell equivalent circuit.

\section{CIRCUIT MODEL FOR TWO COUPLED SLIT ARRAYS UNDER NORMAL INCIDENCE}

In this section the equivalent $\Pi$ circuit for a pair of identical periodic 1D coupled slit gratings is derived. The starting point is given in [40], where fully analytical circuit models were derived for single slit gratings with an electric or magnetic wall placed at a certain distance from the slit array. These problems actually correspond to the odd-even excitation half-problems [62] of the symmetrical coupled grating. This fact is illustrated in Fig. 2, where the rightmost drawing shows a transmission line (representing the fundamental mode propagating in the external region) loaded with appropriate equivalent admittances. These admittances correspond to the input admittances of the slit array printed on a dielectric slab of thickness $d_{1} / 2$ terminated with a magnetic (open-circuit; $Y_{\text {eq }}^{\mathrm{e}}$ ) or an electric (short-circuit; $Y_{\mathrm{eq}}^{\mathrm{o}}$ ) wall. The superscripts "e" and "o" stand for even and odd excitations, respectively.

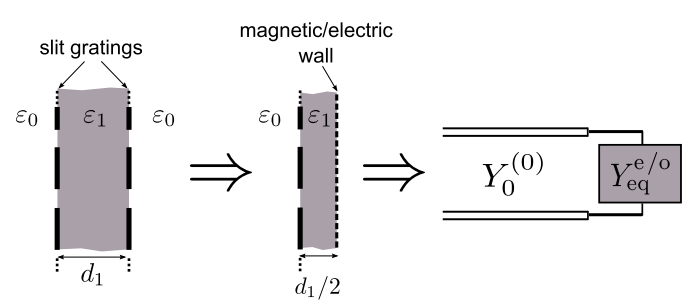

FIG. 2. Even-odd excitation analysis using magnetic or electric walls in the symmetry plane and its circuit representation according to Ref. [40]. The superscript "e" stands for even excitation; the superscript "o," for odd excitation. 
According to [40], $Y_{\mathrm{eq}}^{\mathrm{e}}$ and $Y_{\mathrm{eq}}^{\mathrm{o}}$ are given by the infinite sum of modal admittances,

$$
\begin{aligned}
& Y_{\text {eq }}^{\mathrm{e}}=\sum_{n=1}^{\infty} A_{n} Y_{n}^{(0)}+\mathrm{j} \sum_{n=0}^{\infty} A_{n} Y_{n}^{(1)} \tan \left(\beta_{n}^{(1)} d_{1} / 2\right), \\
& Y_{\text {eq }}^{\mathrm{o}}=\sum_{n=1}^{\infty} A_{n} Y_{n}^{(0)}-\mathrm{j} \sum_{n=0}^{\infty} A_{n} Y_{n}^{(1)} \cot \left(\beta_{n}^{(1)} d_{1} / 2\right),
\end{aligned}
$$

where

$$
\begin{gathered}
A_{n}=\left(2-\delta_{n 0}\right) \times \begin{cases}J_{0}^{2}\left(k_{n} w / 2\right), & \text { TM incidence, } \\
{\left[2 \frac{J_{1}\left(k_{n} w / 2\right)}{k_{n} w / 2}\right]^{2},} & \text { TE incidence, }\end{cases} \\
Y_{n}^{(i)}=\frac{1}{\eta_{0}} \times \begin{cases}\varepsilon_{\mathrm{r}}^{(i)} k_{0} / \beta_{n}^{(i)}, & \text { TM incidence, } \\
\beta_{n}^{(i)} / k_{0}, & \text { TE incidence, }\end{cases} \\
\beta_{n}^{(i)}=\sqrt{\varepsilon_{\mathrm{r}}^{(i)} k_{0}^{2}-k_{n}^{2},} \\
k_{0}=\frac{\omega}{c}, \quad k_{n}=\frac{2 \pi n}{p},
\end{gathered}
$$

where $\delta_{i j}$ is the Kronecker delta, $J_{i}(\cdot)$ the Bessel function of the first kind and $i$ th order, $\eta_{0}=\sqrt{\mu_{0} / \varepsilon_{0}}$ the intrinsic impedance of free space, $\omega$ the angular frequency, $c$ the speed of light in free space, $k_{n}$ the cutoff wave number of the $n$th mode supported by the parallel-plate waveguide associated with the unit cell, and $\beta_{n}^{(i)}$ the corresponding longitudinal wave number of the $n$th mode in medium $(i)$. The $A_{n}$ coefficient is the $n$th Fourier coefficient of the normalized electric-field profile assumed at the slit aperture $(y \in[-w / 2, w / 2]$, with $w$ being the width of the slit), which is taken as

$$
E_{\text {slit }}(y)= \begin{cases}\frac{2}{\pi w}\left[1-(2 y / w)^{2}\right]^{-1 / 2}, & \text { TM incidence, } \\ \frac{4}{\pi w}\left[1-(2 y / w)^{2}\right]^{1 / 2}, & \text { TE incidence }\end{cases}
$$

(note that this approximation accounts for the correct edge behavior of the aperture field in both the TM and the TE cases).

Starting from the basic circuit models for the even- and odd-excitation-half problems shown in Fig. 2, our first goal in Sec. II A is to find an equivalent circuit for the pair of coupled gratings in the form of an equivalent $\Pi$ network. Then Sec. II B presents a detailed study of the elements in the $\Pi$ circuit, leading to a further decomposition into different elements that allows for their efficient computation and provides an insightful physical interpretation. Finally, Sec. IIC presents the strategy proposed for the computation of the П-circuit elements according to the discussion in Sec. II B, and Sec. II D presents various numerical results that serve to illustrate and support the previous discussion as well as to demonstrate the quantitative performance of the $\Pi$-network circuit model.

\section{A. Derivation of the equivalent $\Pi$ network for coupled gratings}

In order to find the equivalent $\Pi$ network that describes the behavior of the coupled screens [see Fig. 3(a)], an evenodd excitation analysis is also applied to this $\Pi$ circuit by placing open- or short-circuit terminations in the middle plane [Fig. 3(b)]. As a result, the half-problem circuits shown in Figs. 3(c) and 3(d) are obtained. Identifying the latter two

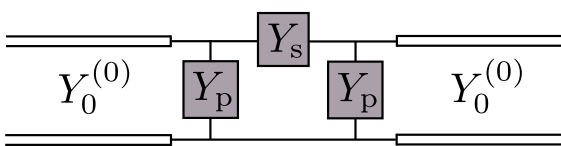

(a)

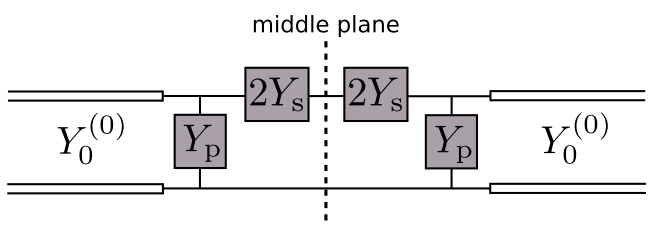

(b)

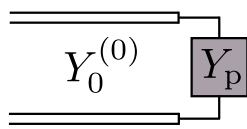

(c)

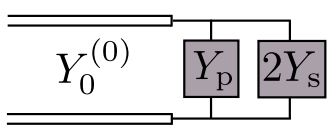

(d)
FIG. 3. (a) Equivalent $\Pi$ circuit for a pair of coupled gratings. (b) The same circuit as in (a), showing the middle symmetry plane. (c, d) The circuits that result from applying open or short circuit terminations (corresponding to even or odd excitations) at the middle symmetry plane.

circuits with the one in Fig. 2, it is clear that the parallel and series admittances $\left(Y_{\mathrm{p}}\right.$ and $\left.Y_{\mathrm{s}}\right)$ can readily be obtained from the even and odd equivalent admittances as

$$
Y_{\mathrm{p}}=Y_{\mathrm{eq}}^{\mathrm{e}}, \quad Y_{\mathrm{s}}=\frac{1}{2}\left[Y_{\mathrm{eq}}^{\mathrm{o}}-Y_{\mathrm{eq}}^{\mathrm{e}}\right],
$$

and therefore, from (1) and (2),

$$
\begin{gathered}
Y_{\mathrm{p}}=\sum_{n=1}^{\infty} A_{n} Y_{n}^{(0)}+\mathrm{j} \sum_{n=0}^{\infty} A_{n} Y_{n}^{(1)} \tan \left(\beta_{n}^{(1)} d_{1} / 2\right), \\
Y_{\mathrm{s}}=-\mathrm{j} \sum_{n=0}^{\infty} A_{n} Y_{n}^{(1)} \csc \left(\beta_{n}^{(1)} d_{1}\right) .
\end{gathered}
$$

It should be noted [57] that the first summation in $Y_{\mathrm{p}}$,

$$
Y_{\mathrm{p}}^{(0)}=\sum_{n=1}^{\infty} A_{n} Y_{n}^{(0)}
$$

only accounts for the external field, whereas the second summation is associated with the field in region (1):

$$
Y_{\mathrm{p}}^{(1)}=\mathrm{j} \sum_{n=0}^{\infty} A_{n} Y_{n}^{(1)} \tan \left(\beta_{n}^{(1)} d_{1} / 2\right) .
$$

This fact is key in Sec. III to obtaining the equivalent circuit for stacked structures involving an arbitrary number of gratings.

\section{B. Interpretation of the admittances deduced for the $\Pi$ circuit}

The analytical expressions found in previous sections for the circuit elements of the $\Pi$ network have been derived as infinite series, and certainly, these formal expressions are not well suited for a qualitative understanding of the behavior of the fields, nor are they convenient from a computational perspective. However, it will be shown that they can be easily manipulated and rewritten in a more insightful and suitable form. First, it is convenient $[40,43]$ to split the infinite series in 
(10), (11), and (12) into two separate contributions. The first contribution comprises the low-order terms, which correspond to propagating modes at the highest frequency of interest. It is also advisable to add the first evanescent mode to this low-order group. The second contribution is formed by an infinite series of evanescent modes whose cutoff frequencies are far above the working frequency, namely, high-order ("ho") evanescent modes. Expressions in (10), (11), and (12) are thus regrouped as

$$
\begin{aligned}
Y_{\mathrm{p}}^{(0)} & =\sum_{n=1}^{N} A_{n} Y_{n}^{(0)}+\underbrace{\sum_{n=N+1}^{\infty} A_{n} Y_{n}^{(0)},}_{Y_{\mathrm{p}, \text { ho }}^{(0)}} \\
Y_{\mathrm{p}}^{(1)}= & \mathrm{j} \sum_{n=0}^{N} A_{n} Y_{n}^{(1)} \tan \left(\beta_{n}^{(1)} d_{1} / 2\right) \\
& +\underbrace{\sum_{n}^{\infty} A_{n} Y_{n}^{(1)} \tan \left(\beta_{n}^{(1)} d_{1} / 2\right)}_{n=N+1}, \\
Y_{\mathrm{s}}= & -\mathrm{j} \sum_{n=0}^{N} A_{n} Y_{n}^{(1)} \csc \left(\beta_{n}^{(1)} d_{1}\right) \\
& -\underbrace{\infty}_{n=N+1} A_{n}^{\infty} Y_{n}^{(1)} \csc \left(\beta_{n}^{(1)} d_{1}\right)
\end{aligned}
$$

where $N$ is the number of low-order ("lo") terms. According to the above-mentioned criterion, $N$ will be given by

$$
N=\left\lceil\sqrt{\varepsilon_{\mathrm{r}, \text { max }}} \frac{p}{\lambda_{0, \text { min }}}\right\rceil,
$$

where $\lceil\cdot\rceil$ represents the smallest following integer (ceiling function), $\varepsilon_{\mathrm{r} \text {,max }}$ is the greater of the two surrounding permittivities, and $\lambda_{0, \text { min }}$ is the minimum vacuum wavelength value of interest. Since the modes that contribute to the $Y_{\mathrm{p}, \mathrm{ho}}^{(i)}$ and $Y_{\mathrm{s} \text {,ho }}$ high-order admittances are far below their cutoff frequencies, the following approximation can be used:

$$
k_{n} \gg \sqrt{\varepsilon_{\mathrm{r}}^{(i)}} k_{0}, \quad \beta_{n, \mathrm{ho}}^{(i)} \approx-\mathrm{j} k_{n} \quad(i=0,1) .
$$

The expressions for the TM and TE characteristic admittances of these modes then reduce to

$$
\begin{array}{ll}
Y_{n, \mathrm{ho}}^{(i)} \approx \mathrm{j} \omega \frac{\varepsilon_{0} \varepsilon_{\mathrm{r}}^{(i)}}{k_{n}}=\mathrm{j} \omega C_{n}^{(i)}, & \text { TM case } \\
Y_{n, \mathrm{ho}}^{(i)} \approx \frac{1}{\mathrm{j} \omega} \frac{k_{n}}{\mu_{0}}=\frac{1}{\mathrm{j} \omega L_{n}}, & \text { TE case },
\end{array}
$$

whose frequency dependence is readily recognized as standard reactive lumped elements (inductors and capacitors). Depending on the polarization of the impinging wave, a lumped $n$-order capacitor $C_{n}^{(i)}$ or $n$-order inductance $L_{n}$ is defined for the $n$ th-order mode (note that $L_{n}$ does not depend on the permittivity of the medium, as expected for a standard inductance).
For TM polarization, introducing (18) in $Y_{\mathrm{p}}^{(i)}$ and $Y_{\mathrm{s}}$, we obtain

$$
\begin{gathered}
Y_{\mathrm{p}, \text { ho }}^{(0)}=\mathrm{j} \omega \sum_{n=N+1}^{\infty} A_{n} C_{n}^{(0)}=\mathrm{j} \omega C_{\mathrm{p}}^{(0)}, \\
Y_{\mathrm{p}, \mathrm{ho}}^{(1)}=\mathrm{j} \omega \sum_{n=N+1}^{\infty} A_{n} C_{n}^{(1)} \tanh \left(k_{n} d_{1} / 2\right)=\mathrm{j} \omega C_{\mathrm{p}}^{(1)}, \\
Y_{\mathrm{s}, \text { ho }}=\mathrm{j} \omega \sum_{n=N+1}^{\infty} A_{n} C_{n}^{(1)} \operatorname{csch}\left(k_{n} d_{1}\right)=\mathrm{j} \omega C_{\mathrm{s}},
\end{gathered}
$$

where $C_{\mathrm{p}}^{(1)}$ and $C_{\mathrm{s}}$ are capacitors that account for the global effect of all the higher-order evanescent modes.

Similarly, for TE polarization the following equivalent inductances associated with the effect of all the higher-order modes are obtained:

$$
\begin{gathered}
Y_{\mathrm{p}, \text { ho }}^{(0)}=\frac{1}{\mathrm{j} \omega} \sum_{n=N+1}^{\infty} A_{n} / L_{n}=\frac{1}{\mathrm{j} \omega L_{\mathrm{p}}^{(0)}}, \\
Y_{\mathrm{p}, \mathrm{ho}}^{(1)}=\frac{1}{\mathrm{j} \omega} \sum_{n=N+1}^{\infty}\left(A_{n} / L_{n}\right) \tanh \left(k_{n} d_{1} / 2\right)=\frac{1}{\mathrm{j} \omega L_{\mathrm{p}}^{(1)}}, \\
Y_{\mathrm{s}, \text { ho }}=\frac{1}{\mathrm{j} \omega} \sum_{n=N+1}^{\infty}\left(A_{n} / L_{n}\right) \operatorname{csch}\left(k_{n} d_{1}\right)=\frac{1}{\mathrm{j} \omega L_{\mathrm{s}}} .
\end{gathered}
$$

The circuit models in the form obtained thus far are depicted in Fig. 4, with the high-order capacitances and inductances explicitly shown. For simplicity, in these drawings it is assumed that $N=0$; i.e., the frequently encountered situation in which only the fundamental zero-order term contributes to the low-order admittances that appear in the internal $\Pi$ network. All the higher-order modes are then included in the lumped elements.

As pointed out for the single-grating problem [40], a more specific physical interpretation of these lumped reactive elements can be found after noting that they represent the

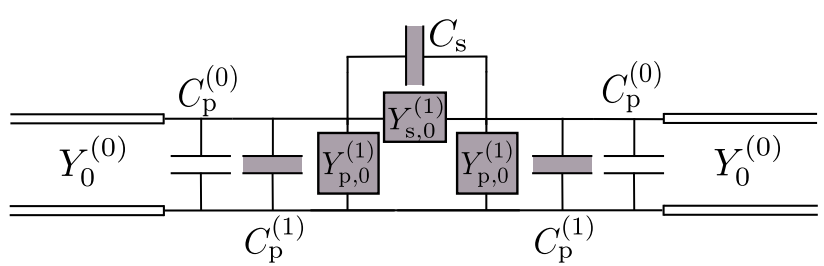

(a)

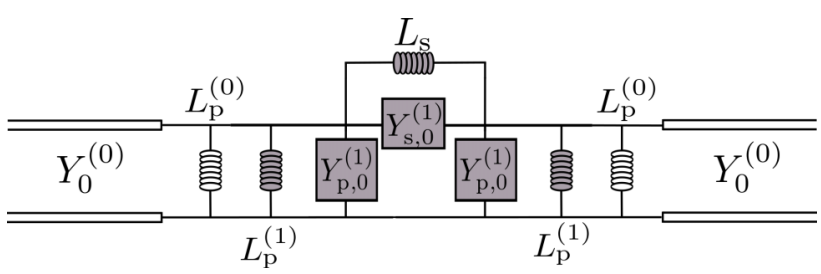

(b)

FIG. 4. (a) Equivalent circuit showing separately the contribution of the fundamental mode ( $\Pi$ network) and the higher-order modes (lumped capacitors and inductors). (a) TM polarization; (b) TE polarization. 
reactive field excited in the vicinity of the slit aperture (since they account for the evanescent modes). This interpretation can directly be translated to the external high-order element $\left(C_{\mathrm{p}}^{(0)}\right.$ or $\left.L_{\mathrm{p}}^{(0)}\right)$, which represents the reactive near field around the slit in the external region. For the internal region it is also desirable to find an interpretation for both the series and the parallel reactive lumped elements. For the series element in (22) or (25), the exponential behavior of the $\operatorname{csch}(\cdot)$ function for large arguments makes this series admittance decrease exponentially as the two coupled screens are separated, which clearly suggests that the series element is basically related to the coupling between both screens through evanescent fields. From (22) or (25) it is apparent that only the evanescent modes that reach the other screen will contribute significantly to the series element, with the contribution of the remaining modes being negligible. On the contrary, the parallel element in (21) or (24) does not vanish for distant screens, since the $\tanh (\cdot)$ function approaches unity instead. Therefore, for coupled screens sufficiently separated (so that the reactive field around the slits does not reach the other screen), the parallel lumped element approaches its single-grating value. This suggests further splitting the parallel element as

$$
C_{\mathrm{p}}^{(1)}=\underbrace{\sum_{n=N+1}^{\infty} A_{n} C_{n}^{(1)}}_{C_{\mathrm{p}, \text { single }}^{(1)}}-\underbrace{\sum_{n=N+1}^{\infty} A_{n} C_{n}^{(1)}\left[1-\tanh \left(k_{n} d_{1} / 2\right)\right] .}_{C_{\mathrm{p}, \text { coup }}}
$$

The first term, $C_{\mathrm{p} \text {, single }}^{(1)}$, represents the value of the reactive element in the absence of coupling with a second screen (single-grating value). The second one, $C_{\mathrm{p}, \text { coup }}$, is a coupling term that accounts for the modification of the single-screen capacitance due to the presence of the other screen. The fact that this term is subtracted from $C_{\mathrm{p} \text {, single indicates }}^{(1)}$ that the overall parallel capacitance tends to decrease as a consequence of the coupling, and this can be interpreted as a parallel connection between the single-screen capacitance and a negative capacitance, $-C_{\mathrm{p} \text {,coup }}$. A similar decomposition can be made for the parallel inductance in the TE case as

$$
1 / L_{\mathrm{p}}^{(1)}=\underbrace{\sum_{n=N+1}^{\infty} A_{n} / L_{n}}_{1 / L_{\mathrm{p}, \text { single }}=1 / L_{\mathrm{p}}^{(0)}}-\underbrace{\sum_{n=N+1}^{\infty} A_{n} / L_{n}\left[1-\tanh \left(k_{n} d_{1} / 2\right)\right]}_{L_{\mathrm{p}, \text { coup }}} .
$$

As found for the series coupling elements in (22) and (25), only those higher-order modes whose evanescent field reaches the other screen need to be included in the parallel coupling elements (26) and (27). A convenient criterion to determine the highest-order mode, $M$, to be considered in the $C_{\mathrm{s}}, L_{\mathrm{s}}$, $C_{\mathrm{p}, \text { coup }}$, and $L_{\mathrm{p}, \text { coup }}$ summations in (22), (25), (26), and (27) is found to be

$$
k_{M} d_{1} \approx 1 \Longrightarrow M=\left\lceil\frac{1}{2 \pi} \frac{p}{d_{1}}\right\rceil .
$$

This criterion has been satisfactorily checked in situations in which coupling through evanescent modes play a significant role.

Finally, in Fig. 4 the contribution of the zero-order mode is accounted for by the internal $\Pi$ circuit formed by the

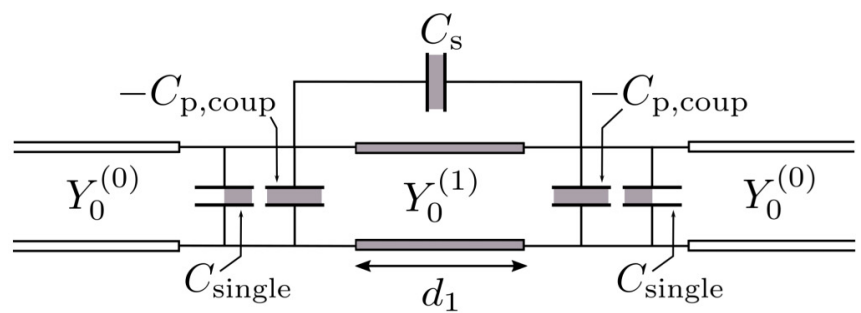

(a)

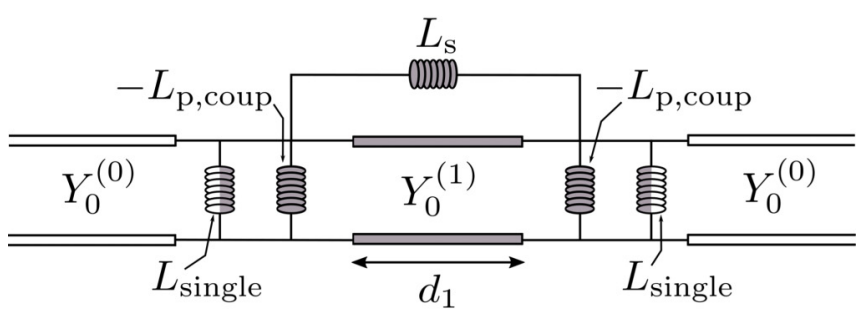

(b)

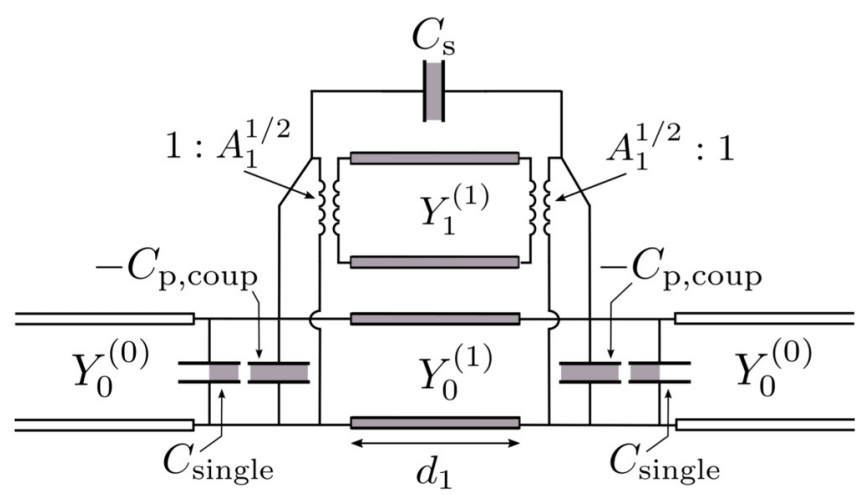

(c)

FIG. 5. Circuit models for (a) TM and (b) TE polarizations considering the fundamental mode as the single distributed element (transmission line of admittance $Y_{0}^{(1)}$ ). The higher-order-mode contribution is included in the lumped elements. (c) Circuit model for the case of two distributed modes (only the TM polarization case).

$Y_{\mathrm{p}, 0}^{(1)}$ and $Y_{\mathrm{s}, 0}$ admittances. After some manipulations (see the Appendix), it can be shown that such a low-order $\Pi$ circuit is equivalent to a transmission-line section with characteristic admittance $Y_{0}^{(1)}$ and length $d_{1}$.

The above ideas are illustrated in the circuits shown in Fig. 5, with the aim of gaining a graphical insightful interpretation (it is again assumed that the zero-order mode is the only low-order term; $N=0$ ). The internal transmission line with admittance $Y_{0}^{(1)}$ accounts for the coupling between the screens through the propagating zero-order wave. The reactive elements labeled "single" represent the reactive field around the slits for a single grating (i.e., in the absence of coupling through evanescent fields). For TM polarization this element is obtained as the sum (parallel connection) of $C_{\mathrm{p}}^{(0)}$ in (20) and $C_{\mathrm{p} \text {,single }}^{(1)}$ in (26) (for TE polarization it is given by the parallel connection between the corresponding inductances). If the two coupled screens are distant enough so that there is not significant coupling through evanescent modes (namely, if $M=0$ for the cases in Figs. 5(a) and 5(b) 
or $M \leqslant N$ in general), then the reactive coupling elements can be neglected. This situation, with only one propagating mode and no reactive coupling, is obtained when the period is electrically small and the dielectric slab is electrically thick. In the literature, many authors restrict themselves to the analysis of such a situation. However, closely spaced gratings are important for many practical cases of interest, and in such cases, the coupling through evanescent fields should actually be taken into account, which can conveniently be carried out by introducing the series and parallel coupling capacitors or inductors. Furthermore, in order to extend the range of applications of the model to higher frequencies where one or more higher-order modes become propagating in the slab between the gratings, the full dynamic frequency dependence of their modal admittances and propagation wave numbers has to be considered. As $N>0$, these higher-order modes must contribute to the low-order sums in (13), (14), and (15) and its respective effect can similarly be separated from the high-order elements to form another $\Pi$ circuit, which is again equivalent to another transmission line with its corresponding admittance $Y_{i}^{(1)}$ and length $d_{1}$. This fact is sketched in Fig. 5(c) for $N=1$ and TM polarization. The appearance of transformers of turn ratio $A_{1}^{1 / 2}$ placed at both ends of the transmission line is noteworthy.

\section{Strategy for the computation of the П-circuit admittances}

Taking into account the ideas discussed above, we now discuss the systematic strategy followed in this work to compute the $Y_{\mathrm{p}}^{(0)}, Y_{\mathrm{p}}^{(1)}$, and $Y_{\mathrm{s}}$ admittances that make up the $\Pi$ circuit. First, given the structural parameters and frequency range of interest, the value of $N$ is computed following the criterion proposed in (16). Using this value of $N$, the $C_{\mathrm{p}}^{(0)}$ and $C_{\mathrm{p}, \text { single }}^{(1)}$ lumped elements in (20) and (26) are computed as

$$
\begin{gathered}
C_{\mathrm{p}}^{(0)}=w \varepsilon_{0} \varepsilon_{\mathrm{r}}^{(0)} \sum_{n=N+1}^{\infty} \frac{\left[J_{0}\left(k_{n} w / 2\right)\right]^{2}}{k_{n} w / 2}, \\
C_{\mathrm{p}, \text { single }}^{(1)}=\frac{\varepsilon_{\mathrm{r}}^{(1)}}{\varepsilon_{\mathrm{r}}^{(0)}} C_{\mathrm{p}}^{(0)} .
\end{gathered}
$$

Similarly, for the TE case, the $L_{\mathrm{p}}^{(0)}$ and $L_{\mathrm{p} \text {,single }}^{(1)}$ elements in (23) and (27) are obtained from

$$
\frac{1}{L_{\mathrm{p}}^{(0)}}=\frac{1}{L_{\mathrm{p}, \text { single }}^{(1)}}=\frac{16}{w \mu_{0}} \sum_{n=N+1}^{\infty} \frac{\left[J_{1}\left(k_{n} w / 2\right)\right]^{2}}{k_{n} w / 2} .
$$

Next, the value of $M$ is obtained from the criterion in (28). If $M>N$, then the coupling reactive elements are computed as

$$
\begin{aligned}
C_{\mathrm{p}, \text { coup }} & =w \varepsilon_{0} \varepsilon_{\mathrm{r}}^{(1)} \sum_{n=N+1}^{M} \frac{\left[J_{0}\left(k_{n} w / 2\right)\right]^{2}}{k_{n} w / 2}\left[1-\tanh \left(k_{n} d_{1} / 2\right)\right], \\
C_{\mathrm{s}} & =w \varepsilon_{0} \varepsilon_{\mathrm{r}}^{(1)} \sum_{n=N+1}^{M} \frac{\left[J_{0}\left(k_{n} w / 2\right)\right]^{2}}{k_{n} w / 2} \operatorname{csch}\left(k_{n} d_{1}\right)
\end{aligned}
$$

or

$$
\begin{gathered}
\frac{1}{L_{\mathrm{p}, \text { coup }}}=\frac{16}{w \mu_{0}} \sum_{n=N+1}^{M} \frac{\left[J_{1}\left(k_{n} w / 2\right)\right]^{2}}{k_{n} w / 2}\left[1-\tanh \left(k_{n} d_{1} / 2\right)\right], \\
\frac{1}{L_{\mathrm{s}}}=\frac{16}{w \mu_{0}} \sum_{n=N+1}^{M} \frac{\left[J_{1}\left(k_{n} w / 2\right)\right]^{2}}{k_{n} w / 2} \operatorname{csch}\left(k_{n} d_{1}\right) .
\end{gathered}
$$

If $M \leqslant N$, these coupling elements are ignored. Once all of the above lumped elements are known, the high-order admittances in (13)-(15) are given by

$$
\begin{gathered}
Y_{\mathrm{p}, \mathrm{ho}}^{(0)}=\mathrm{j} \omega C_{\mathrm{p}}^{(0)}, \\
Y_{\mathrm{p}, \mathrm{ho}}^{(1)}=\mathrm{j} \omega\left(C_{\mathrm{p}, \text { single }}^{(1)}-C_{\mathrm{p}, \text { coup }}\right), \\
Y_{\mathrm{s}, \mathrm{ho}}=\mathrm{j} \omega C_{\mathrm{s}},
\end{gathered}
$$

and the corresponding expressions for the TE case are obtained by replacing $\mathrm{j} \omega C$ with $1 / \mathrm{j} \omega L$. It is important to note that none of the above lumped elements depends on frequency. Hence, they have to be evaluated only once when performing a frequency sweep. Furthermore, the elements with infinite summations in (29)-(31) do not depend on $d_{1}$ either, and therefore it is not necessary to recalculate them when varying the distance between the gratings, provided the period $p$ or the slit width $w$ is not modified. If so desired, diverse techniques can be applied to find closed-form expressions for the involved series [23]. More specifically, all the numerical series required in this paper, which involve Bessel functions in their general term, have been computed in closed form in the frame of the analysis of the so-called boxed microstrip line $[65,66]$. Thus, the results in [65] and [66] can be directly employed to speed up the computation of the series in the present work. Finally, for each value of frequency, the admittances in the $\Pi$ circuit are obtained as

$$
\begin{gathered}
Y_{\mathrm{p}}^{(0)}=\sum_{n=1}^{N} A_{n} Y_{n}^{(0)}+Y_{\mathrm{p}, \mathrm{ho}}^{(0)}, \\
Y_{\mathrm{p}}^{(1)}=\mathrm{j} \sum_{n=0}^{N} A_{n} Y_{n}^{(1)} \tan \left(\beta_{n}^{(1)} d_{1} / 2\right)+Y_{\mathrm{p}, \mathrm{ho}}^{(1)}, \\
Y_{\mathrm{S}}=-\mathrm{j} \sum_{n=0}^{N} A_{n} Y_{n}^{(1)} \csc \left(\beta_{n}^{(1)} d_{1}\right)+Y_{\mathrm{s}, \mathrm{ho}} .
\end{gathered}
$$

\section{Results and discussion}

Some numerical results are next presented in order to check the validity and accuracy of the equivalent-circuit approach for a pair of coupled gratings and to illustrate the previous discussion. Figure 6 shows the transmission coefficient of a pair of coupled gratings printed on either side of a dielectric slab, for two values of the slab thickness $d_{1}$ (separation between the gratings).

For comparison purposes, we have developed an in-house numerical code based on the method of moments (MoM) solution of the integral equation for the aperture field. This MoM implementation, which uses several basis functions to 


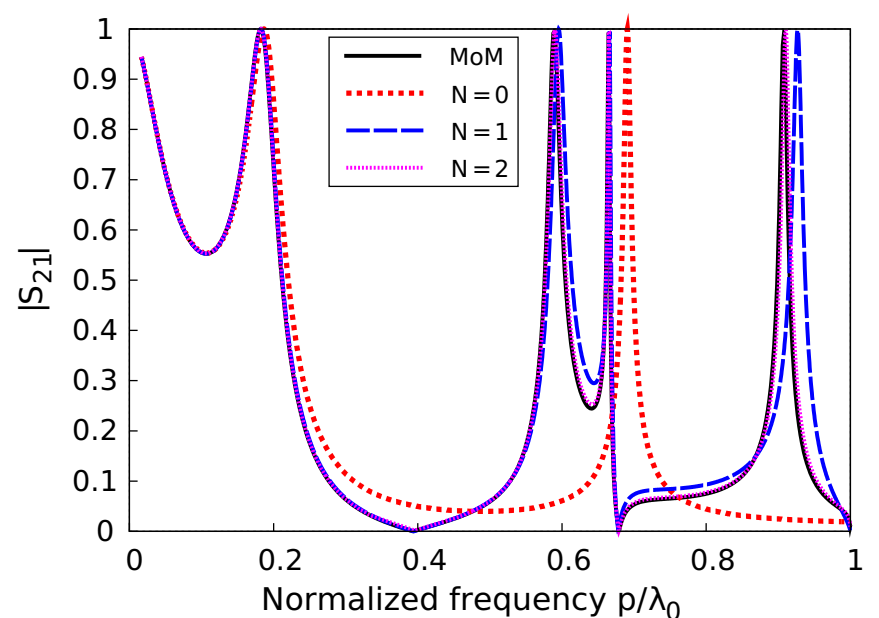

(a)

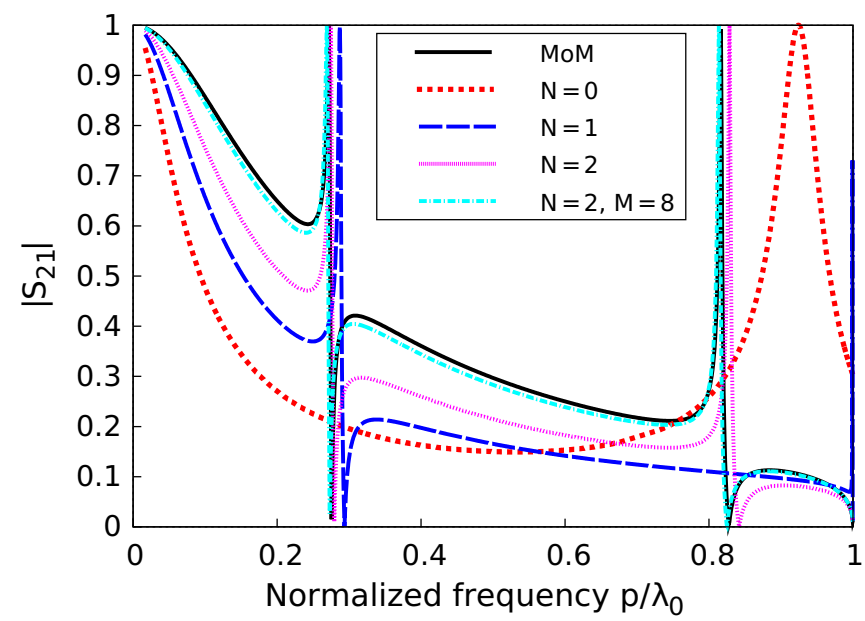

(b)

FIG. 6. Magnitude of the transmission coefficient $\left(\left|S_{21}\right|\right)$ for two coupled gratings versus the normalized frequency for TM incidence and for different thicknesses $\left(d_{1}\right)$ of the dielectric slab between the gratings (i.e., for different values of the separation between the gratings). Parameters: $w=0.1 p, \varepsilon_{\mathrm{r}}^{(0)}=1, \varepsilon_{\mathrm{r}}^{(1)}=4$. (a) Relatively distant gratings: $d_{1}=p / 2.5=4 w$. (b) Closely spaced gratings: $d_{1}=p / 50=w / 5$.

reproduce the aperture field, has been carefully checked and the numerical results it provides can be considered virtually exact for our purposes.

In the first case shown in Fig. 6(a), the separation between the metallic screens is relatively large (the slab thickness is 2.5 times smaller than the periodicity of the slit gratings). Indeed, the value of the ratio $p /\left(2 \pi d_{1}\right)$ is quite low $(\approx 0.4)$, and consequently, the coupling through evanescent fields is not expected to be relevant. Hence, the $C_{\mathrm{p} \text {,coup }}$ and $C_{\mathrm{s}}$ capacitors do not have to be considered in the circuit model [in other words, $M=0$ is taken for the circuit-model results shown in Fig. 6(a), although $M=1$ could be taken according to our more conservative general criterion given in (28)]. It is shown in the figure that the circuit-model results obtained with $N=0$ reproduce accurately the transmission spectrum at low frequencies, but they tend to deviate as the frequency increases until they become qualitatively wrong. The reason is that the first higher-order mode becomes propagating in the dielectric slab at $p / \lambda_{0}=0.5$. Thus, the $N=0$ results start to deviate at lower frequencies $\left(p / \lambda_{0} \gtrsim 0.2\right)$ where the $n=1$ evanescent mode is no longer far below its cutoff frequency and its increasingly dynamic behavior starts to have an effect on the transmission spectrum. The circuit model can accommodate this situation simply by setting $N=1$, i.e., by incorporating the corresponding $\Pi$ network for the $n=1$ mode (together with that for the fundamental $n=0$ wave, which is always present). This is confirmed by the $N=1$ results in Fig. 6(a), which show a very good agreement with the MoM curve up to $p / \lambda_{0} \gtrsim 0.6$. For higher frequencies, these results show some deviation with respect to the MoM data, although they are still able to reproduce the main features of the spectrum. Once again, this deviation is due to the fact that the next-higher-order mode $(n=2)$ is approaching its cutoff frequency inside the dielectric slab $\left(p / \lambda_{0}=1\right)$. The circuit model with $N=2$ does provide very accurate results over the whole frequency range.

Next, Fig. 6(b) shows the transmission spectrum for the same two coupled slit gratings as in Fig. 6(a), but now with a much thinner dielectric slab $\left(d_{1}=p / 50\right)$, and hence, the gratings are tightly coupled. Two total transmission peaks are observed, each one followed by a transmission zero. If only the zero-order wave is considered as distributed element in the model through its corresponding $\Pi$ network (curve labeled $N=0$ ), the resulting equivalent circuit is not able to reproduce the transmission behavior at all. When the first higher-order mode is considered a "low-order" mode and thus its dynamic distributed nature is taken into account through the corresponding $\Pi$ network ( $N=1$ curve), the first transmission peak and zero are captured by the circuit model, though slightly blue-shifted. The inclusion of the second higher-order mode $(N=2)$ mostly corrects this frequency shift and makes the proposed circuit model able to reproduce the second peak and zero. However, even with $N=2$ the results are not satisfactory, since a significant difference in the transmission level with respect to MoM results is observed throughout the considered frequency range. The reason is that the circuit used to compute the $N=0, N=1$, and $N=2$ curves in Fig. 6(b) does not include the $C_{\mathrm{p} \text {,coup }}$ and $C_{\mathrm{s}}$ coupling capacitors, which implies that the coupling through evanescent modes is being neglected. As the gratings are now very close to one another, this coupling is expected to be significant. Indeed, for the present configuration and according to our criterion in (28), $M$ should be set to 8 . This means that, for the circuit model with $N=2$, higher-order evanescent modes from $n=3$ to $n=8$ should contribute to the $C_{\mathrm{p} \text {,coup }}$ and $C_{\mathrm{s}}$ capacitors. When these capacitors are also introduced in the circuit model (curve labeled $N=2, M=8$ ), the agreement with the MoM results is very good over the whole frequency range shown. At this point, it is also worth highlighting the computational efficiency of the proposed equivalent-circuit method. Even in this quite stringent case (with three distributed modes and up to eight modes contributing to the evanescent coupling), the CPU time needed for the computation of the $\approx 1000$ values represented in Fig. 6(b) is almost negligible (it takes about $100 \mathrm{~ms}$ in a modest personal computer).

A second example is given in Fig. 7, which shows a comparison between our equivalent-circuit results and experimental results reported in Ref. [54]. The transmissivity coefficient 


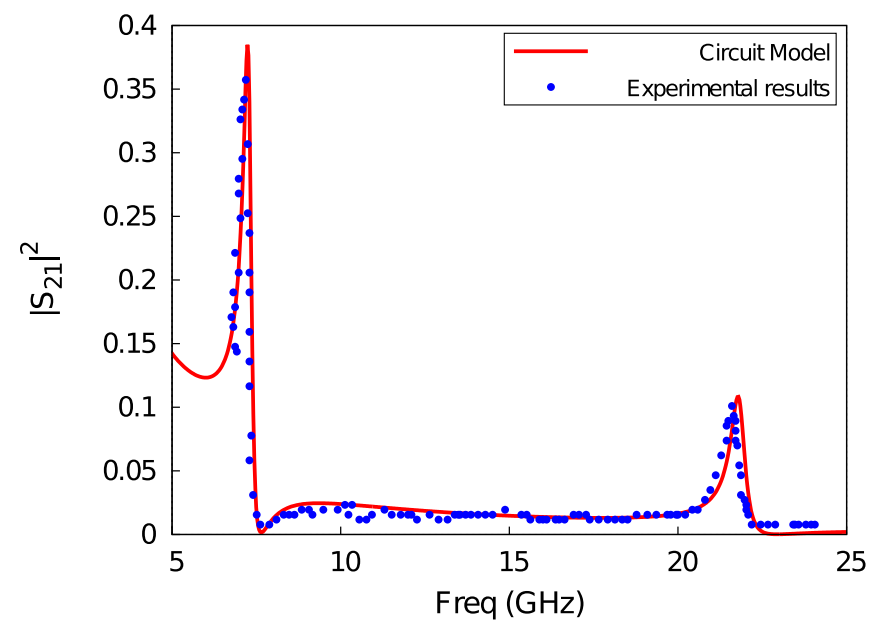

FIG. 7. Comparison of our circuit approach data with experimental results [54] for the transmissivity coefficient $\left(\left|S_{21}\right|^{2}\right)$ of a pair of tightly coupled 1D gratings when a TM-polarized normally incident plane wave impinges on the structure. Parameters: $p=$ $10.02 \mathrm{~mm}, w=0.29 \mathrm{~mm}, d_{1}=0.356 \mathrm{~mm}, \varepsilon_{\mathrm{r}}^{(0)}=1, \varepsilon_{\mathrm{r}}^{(1)}=4.17$, $\tan (\delta)=0.0167$.

is plotted versus the frequency when a normally incident TM plane wave impinges on a pair of coupled arrays. The gratings are printed on the two sides of an electrically very thin and lossy FR4 substrate. It should be pointed out that losses in the substrate can be straightforwardly incorporated in the circuit model through the admittances, (4), and wave numbers, (5), just by replacing the lossless real value of permittivity, $\varepsilon_{r}^{(i)}$, with its complex value for lossy substrates, $\varepsilon_{\mathrm{r}}^{(i)}\left(1-\mathrm{j} \tan \delta^{(i)}\right)$. According to our general criteria in (16) and (28), the circuit-model results in Fig. 7 are computed with $N=2, M=5$. As can be observed, the agreement with the experimental results is remarkably good.

The case of TE incidence is studied in Fig. 8, which shows the frequency behavior of the transmission coefficient when a normally incident plane wave impinges on a pair

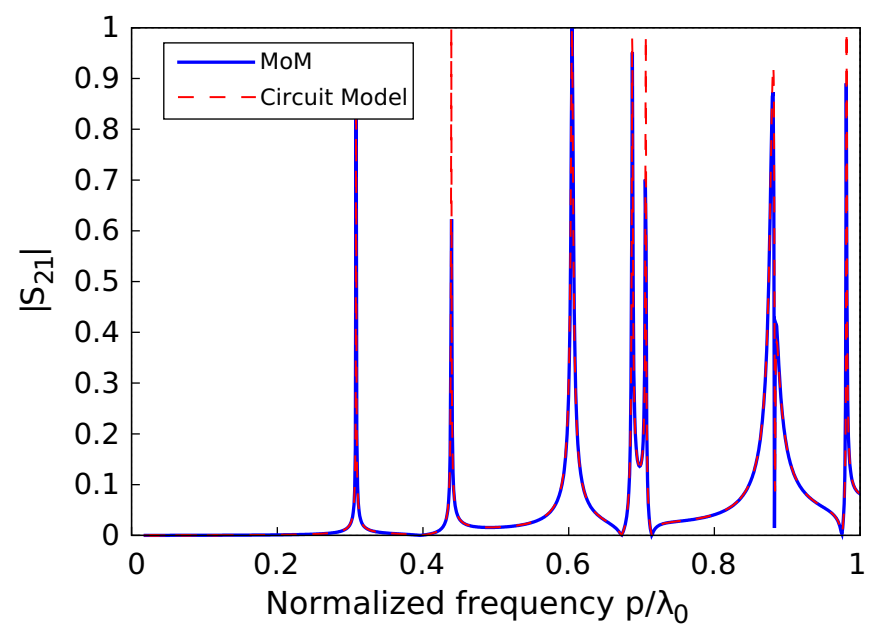

FIG. 8. Transmission coefficient (magnitude) versus frequency for TE incidence. Parameters: $w=0.2 p, d_{1}=0.5 p, \varepsilon_{\mathrm{r}}^{(0)}=1, \varepsilon_{\mathrm{r}}^{(1)}=$ 9.8 . of coupled gratings. For this polarization the interaction of the incident wave with the slits is weaker than for the TM case and an electrically thick medium is needed to observe transmission peaks, which are extremely frequency selective. Four low-order terms were taken as distributed elements $(N=4)$ because of the high permittivity of the substrate. These terms are associated with propagating modes in the dielectric region since the interaction through evanescent modes is almost negligible because the screens are far enough to be affected by them $(M=0)$. Once again, the accuracy shown by the circuit model compared with the numerical MoM data is very good.

Concerning the validity limits of the approach, it is the assumption of a frequency-independent field profile at the slits, (7), that constitutes the fundamental limitation in the application of the model. Therefore, the model is valid for relatively narrow slits; i.e., the results provided by the equivalent circuit will start to deteriorate for frequencies at which the slits are not electrically small. In order to qualitatively estimate the limits of validity, we have carried out extensive numerical experiments for different configurations under both polarizations. For TM polarization, the model is found to provide reliable results for $w / \lambda_{\text {diel }} \lesssim 0.4$, where $\lambda_{\text {diel }}$ is the wavelength inside the highest-permittivity dielectric involved. Under TE polarization, the numerical experiments showed that the validity limit can be estimated as $w / \lambda_{\text {eff }} \lesssim$ 0.75 , where $\lambda_{\text {eff }}$ is the wavelength inside an effective medium with relative permittivity $\varepsilon_{\text {eff }}=\left(\varepsilon_{\mathrm{r}}^{(0)}+\varepsilon_{\mathrm{r}}^{(1)}\right) / 2$.

\section{STACKED ARRAYS}

The previous section presented the derivation of the Пcircuit model for a pair of coupled slit gratings, together with a detailed in-depth physical interpretation of its elements. All the previous derivations and discussions will be found to be very pertinent for the study of stacked arrays since its corresponding equivalent circuit will be systematically built up from the $\Pi$-circuit model for a pair of coupled slit gratings. As already pointed out in Sec. II A, the key fact is that the $Y_{\mathrm{p}}$ admittance in the $\Pi$ network is given by the sum (parallel connection) of the external, $Y_{\mathrm{p}}^{(0)}$, and internal, $Y_{\mathrm{p}}^{(1)}$, parallel admittances. Since these admittances independently account for the field in medium (0) (external medium) and medium (1) (internal medium), an "internal" $\Pi$ block associated with medium (1), characterized by $Y_{\mathrm{p}}^{(1)}$ and $Y_{\mathrm{s}}$, can be defined as shown in Fig. 9. This internal $\Pi$ circuit can be employed as a "building block" to build up the equivalent circuit of a series of stacked arrays, as shown in Fig. 10. The stacked structure amenable to analysis using this procedure may have

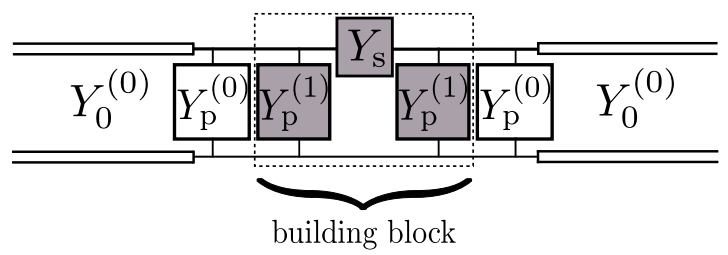

FIG. 9. Topology of the equivalent circuit defining the internal $\Pi$ circuit (building block) and the external part. 


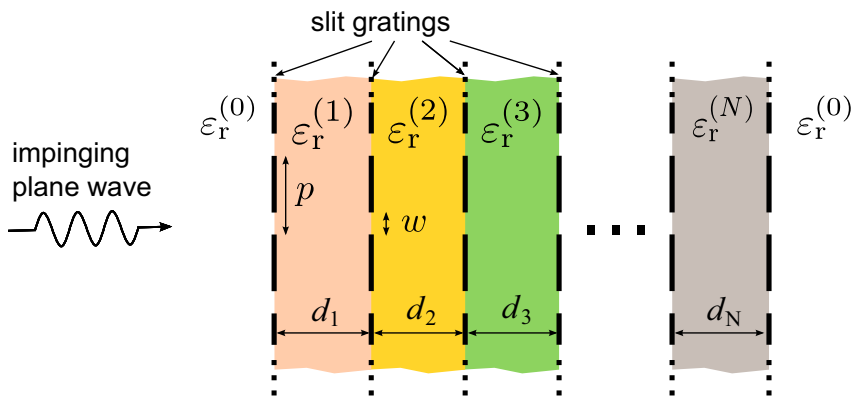

(a)

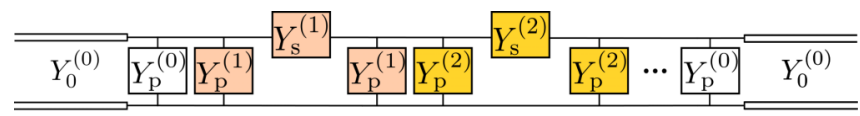

(b)

FIG. 10. (a) Stacked structure with different dielectric slabs. (b) Equivalent circuit made by cascading $\Pi$ building blocks.

dielectric layers of different thickness and permittivity, but the slit arrays must be identical and aligned among them to preserve the periodicity in the transverse direction. It should be noted that the employed strategy is applicable to finite stacked structures thanks to the initial "aperture" formulation of the problem, which made it possible to define two independent subproblems at both sides of the apertures (slits).

The above rationale can also be employed to study an infinite set of periodically stacked slit gratings. In this case, the equivalent network of this longitudinally periodic structure is formed by the periodic repetition of the corresponding $\Pi$ building block (the "unit cell" of the structure along the longitudinal $z$ direction), as illustrated in Fig. 11. As the elements of this $\Pi$ building block are known in closed form, the dispersion relation of the Bloch modes of the infinite

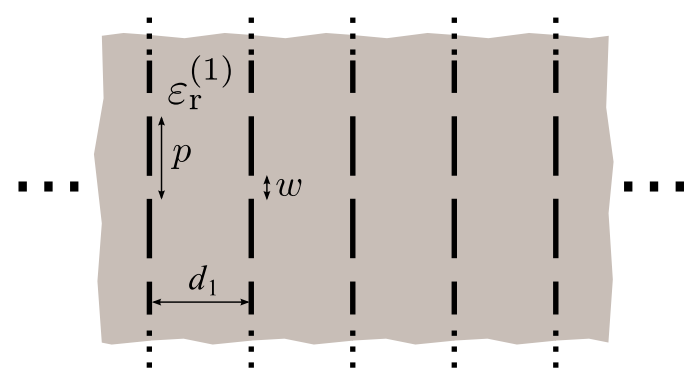

(a)

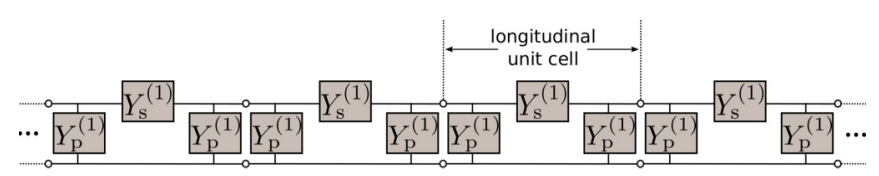

(b)

FIG. 11. (a) Infinite periodic stack of slit gratings. (b) Equivalent circuit consisting of an infinite cascade of $\Pi$ building blocks.

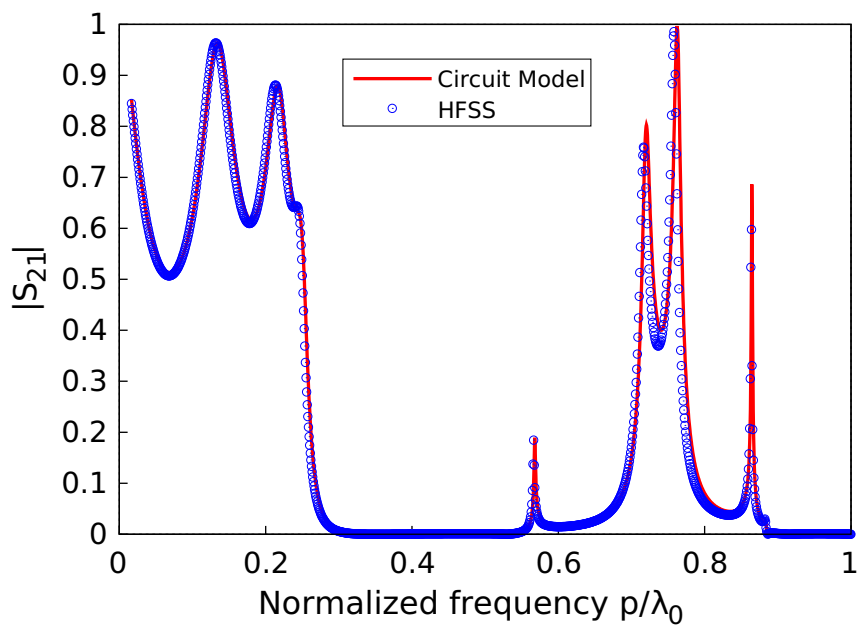

FIG. 12. Magnitude of the transmission coefficient $\left(\left|S_{21}\right|\right)$ versus the normalized frequency for four stacked slit arrays under normal TM incidence. Parameters: $w=0.1 p, d_{1}=0.4 p, d_{2}=0.3 p, d_{3}=$ $0.2 p, \varepsilon_{\mathrm{r}}^{(0)}=1, \varepsilon_{\mathrm{r}}^{(1)}=2.2, \varepsilon_{\mathrm{r}}^{(2)}=4, \varepsilon_{\mathrm{r}}^{(3)}=3$.

periodic stack is readily obtained as [62]

$$
\cosh \left(\gamma d_{1}\right)=1+\frac{Y_{\mathrm{p}}}{Y_{\mathrm{s}}},
$$

where $\gamma=\alpha+\mathrm{j} \beta$ is the complex propagation constant of the Bloch mode, with $\beta$ being the phase constant and $\alpha$ the attenuation constant. The Bloch admittance at the longitudinal unit cell terminals is also derived immediately as [62]

$$
Y_{\mathrm{B}}=\sqrt{Y_{\mathrm{p}}\left(Y_{\mathrm{p}}+2 Y_{\mathrm{s}}\right)}
$$

\section{A. Results and discussion}

As a first example to check the validity of the equivalentcircuit approach for stacked arrays, the transmission coefficient of a set of four cascaded arrays printed on three dielectric slabs under normal TM incidence is plotted in Fig. 12. A high reflection behavior is observed at the center of the plot, from $0.25 p / \lambda_{0}$ up to about $0.7 p / \lambda_{0}$. At low frequencies, a high level of transmission is expected because the slit gratings interact weakly with the incident wave. Additional transmission peaks are also found between $0.7 p / \lambda_{0}$ and $0.9 p / \lambda_{0}$. For comparison purposes, the results provided by the commercial simulator Ansys HFSS [67] (based on the finite-element method in the frequency domain) are also shown. An excellent agreement is found between the two sets of data.

Next, the case of an infinite periodic stack of identical gratings is studied in Fig. 13. The Brillouin diagram for the Bloch mode of this periodic structure obtained from (42) is plotted in Fig. 13(a), which shows four passbands separated by three stopbands. It is interesting to note that the slope of the dispersion relation is positive in the first two passbands (often called forward passbands), whereas it is negative in the two higher-frequency bands (backward passbands). Since no losses are assumed in any element of the present structure, the second member of (42) is always real, providing two possibilities for 


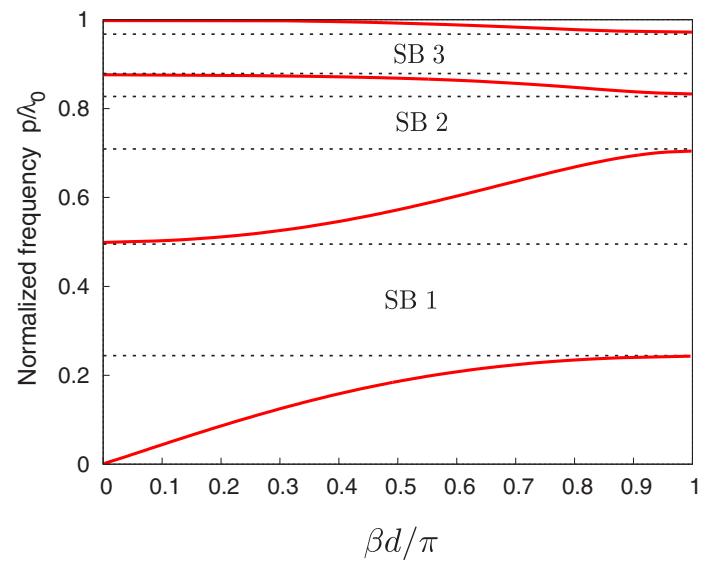

(a)

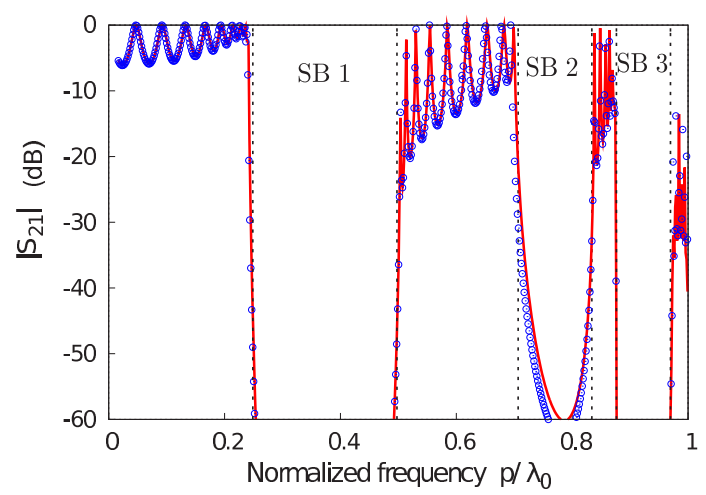

(b)

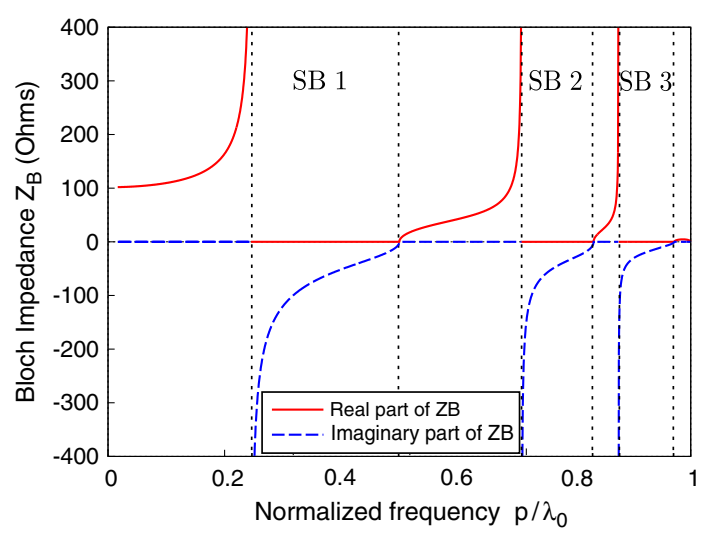

(c)

FIG. 13. (a) Brillouin diagram of an infinite stacked structure. (b) Transmission coefficient $(\mathrm{dB})$ versus normalized frequency for a truncated version (10 stacked gratings). (c) Bloch impedance of the Bloch mode represented in (a). The parameters of the unit cell are $w=0.15 p, d_{1}=0.3 p, \varepsilon_{\mathrm{r}}^{(0)}=1, \varepsilon_{\mathrm{r}}^{(1)}=4$. Frequency regions labeled SB 1, SB 2, and SB 3 denote stopbands.

the propagation constant:

$$
\begin{gathered}
\gamma=\mathrm{j} \beta \quad \text { if } \quad\left[1+\frac{Y_{\mathrm{p}}}{Y_{\mathrm{s}}}\right] \in[-1,1], \\
\gamma=\alpha+\mathrm{j}\left\{\begin{array}{l}
0 \\
\pi d
\end{array} \quad \text { if }\left[1+\frac{Y_{\mathrm{p}}}{Y_{\mathrm{s}}}\right] \notin[-1,1] .\right.
\end{gathered}
$$

Clearly, passbands occur when $\alpha=0(\gamma=\mathrm{j} \beta)$, and stopbands when $\alpha \neq 0$ and $\beta=0$ or $\pi d$. The existence of a passband is then subject to the following two conditions: (i) the reactive nature (capacitive/inductive) of $Y_{\mathrm{s}}$ and $Y_{\mathrm{p}}$ has to be different in order to satisfy $Y_{\mathrm{p}} / Y_{\mathrm{s}}<0$, and (ii) $\left|Y_{\mathrm{p}}\right|<2\left|Y_{\mathrm{s}}\right|$. For instance, these conditions are satisfied if the $\Pi$ network has a relatively small capacitance as a parallel element (close to an open circuit) and a low inductance as a series element (close to a short circuit), which allows the power to pass through the structure, thus obtaining a forward band [62]. On the other hand, the above two conditions can also be satisfied if $Y_{\mathrm{s}}$ is capacitive and $Y_{\mathrm{p}}$ inductive; for instance, a $\Pi$ network with a high capacitance as a series element and a high inductance as the parallel element. This would also allow the power to travel along the stacked structure, but now the passband would be of a backward nature.

If a finite (truncated) version of the previous longitudinally periodic structure is now considered, a clear correspondence is expected between the Brillouin diagram and the behavior of the transmission coefficient of the finite stack. This fact is illustrated in Fig. 13(b), which shows the transmission coefficient through a finite stack with 10 gratings. The presence of successive passbands or stopbands that perfectly correlate with the associated Brillouin diagram in Fig. 13(a) can be observed. The circuit-model results (solid lines) are compared with those provided by the Ansys HFSS (circles), showing an excellent agreement. Another feature of this transmission behavior worthy of mention is the high level of ripple observed in the passbands. This is due to impedance mismatching between the impinging wave (whose characteristic impedance is the intrinsic impedance of the free space, $\eta_{0} \approx 377 \Omega$ ) and the Bloch mode of the stack. Figure 13(c) shows the real and imaginary parts of the Bloch impedance $\left(Z_{\mathrm{B}}=1 / Y_{\mathrm{B}}\right)$ obtained from (43). Since the considered structure is lossless, $Z_{\mathrm{B}}$ is real-valued at passband and purely imaginary at stopband frequencies. For the most part of the first three passbands, the $Z_{\mathrm{B}}$ value is much lower than the free-space impedance, although it tends to grow within each band, which is consistent with the decreasing trend of the ripples. Also, $Z_{\mathrm{B}}$ is higher in the first (low-frequency) passband, and hence the ripples in this band are smaller than in the next two passbands. Finally, the last passband (at the higher frequencies) is the one showing by far the highest mismatching, due to the particularly low value of $Z_{\mathrm{B}}$ at these frequencies.

As the last example, in Fig. 14(a) a lossy stack with the space between the gratings filled with resistive silicon slabs is studied. The complex effective permittivity of the slabs is given by $\varepsilon^{(1)}=\varepsilon_{0} \varepsilon_{\mathrm{r}}^{(1)}-\mathrm{j} \sigma / \omega$, where $\sigma$ is the dc conductivity of the layers. Figure 14(a) shows the power absorbed in a finite stack (normalized to the incident power), whereas the Bloch impedance of the corresponding infinite stack is plotted in Fig. 14(b). Since the structure is lossy, the Bloch impedance takes complex values at any frequency. The most interesting feature is the high-absorption band at high frequencies, with a fractional bandwidth of $4.5 \%$ for a central frequency of 22.5 GHz. As shown in Fig. 14(b), this absorption band can be related to a Bloch impedance with a relatively constant real part that is close to the free-space impedance and with a relatively small imaginary part in the same frequency range. This behavior of the Bloch impedance has been obtained by 


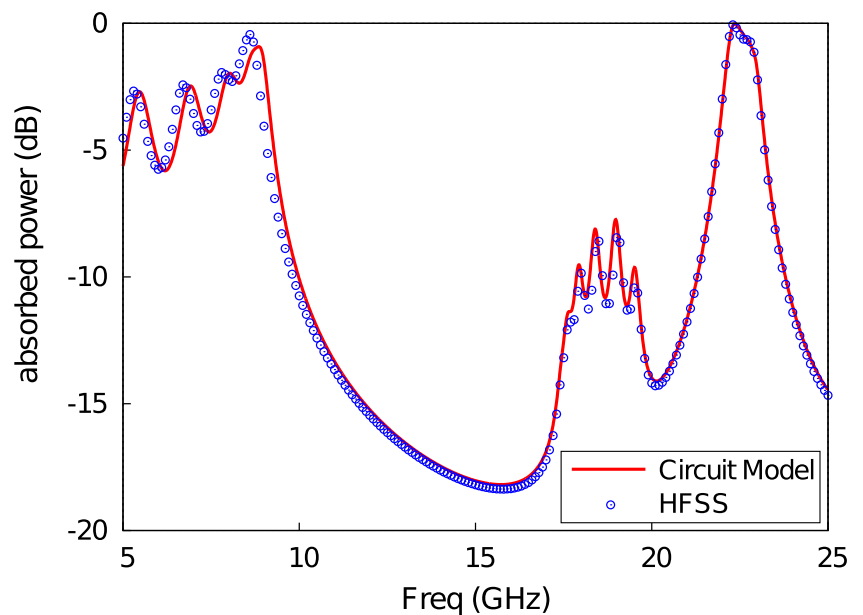

(a)

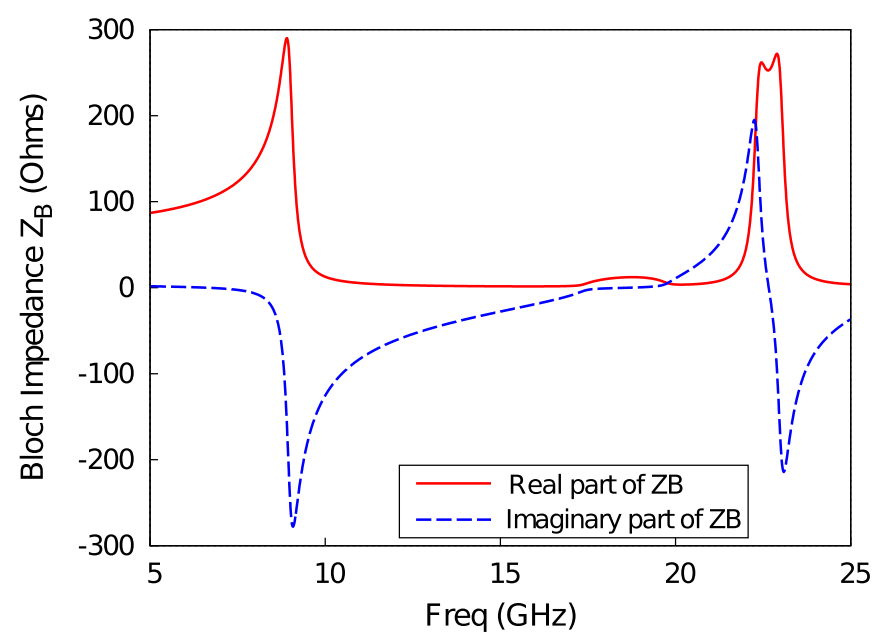

(b)

FIG. 14. (a) Power absorbed in a lossy system formed by eight stacked arrays separated by seven resistive (ohmic) dielectric slabs $(\sigma=0.2 \mathrm{~S} / \mathrm{m})$. (b) Bloch impedance versus frequency. Parameters: $p=5 \mathrm{~mm}, w=1.4 \mathrm{~mm}, d_{1}=2.2 \mathrm{~mm}, \varepsilon_{\mathrm{r}}^{(0)}=1, \varepsilon_{\mathrm{r}}^{(1)}=11.9$.

properly adjusting the geometrical parameters and the value of the slab conductivity. Note that, for frequencies between approximately 17 and $20 \mathrm{GHz}, Z_{\mathrm{B}}$ is mostly real-valued as well. However, its small value implies a strong mismatch with the incident wave that causes most of the incident power to be reflected rather than absorbed in the resistive silicon slabs.

\section{CIRCUIT MODEL FOR OBLIQUE INCIDENCE}

The extension of the circuit model to the case of a plane wave that impinges obliquely with an incident angle $\theta$ is straightforward. Assuming that the incidence is in the $y z$ plane (see Fig. 1), the corresponding incident wave vector is given by

$$
\mathbf{k}=\sqrt{\varepsilon_{\mathrm{r}}^{(0)}} k_{0}(\cos \theta \hat{\mathbf{z}}+\sin \theta \hat{\mathbf{y}}) .
$$

The parallel and series admittances obtained in Sec. II are defined in terms of an infinite sum of modes. For oblique incidence, the transverse boundary conditions of the unit cell are no longer electric or magnetic walls but periodic walls, which implies that the series of modes now become series of Floquet spatial harmonics. Thus, the series and parallel admittances in Fig. 9 can be written as

$$
\begin{gathered}
Y_{\mathrm{p}}^{(0)}=\sum_{\substack{n=-\infty \\
n \neq 0}}^{\infty} A_{n} Y_{n}^{(0)}, \\
Y_{\mathrm{p}}^{(1)}=\sum_{n=-\infty}^{\infty} A_{n} Y_{n}^{(1)} \tan \left(\beta_{n}^{(1)} d_{1} / 2\right), \\
Y_{\mathrm{s}}=\sum_{n=-\infty}^{\infty} A_{n} Y_{n}^{(1)} \csc \left(\beta_{n}^{(1)} d_{1}\right),
\end{gathered}
$$

where

$$
\begin{gathered}
\beta_{n}^{(i)}=\sqrt{\varepsilon_{\mathrm{r}}^{(i)} k_{0}^{2}-\left(k_{n}+k_{\mathrm{t}}\right)^{2}}, \\
k_{t}=\sqrt{\varepsilon_{\mathrm{r}}^{(0)}} k_{0} \sin (\theta),
\end{gathered}
$$

with $k_{t}$ being the transverse (to $z$ ) wave number. For the definition of the $A_{n}$ coefficients, the same field profiles as in (7) are considered, and therefore their expressions are similar to those in (3) but now incorporate the phase shift imposed by the incident plane wave:

$$
A_{n}= \begin{cases}{\left[J_{0}\left(\left(k_{n}+k_{\mathrm{t}}\right) w / 2\right)\right]^{2},} & \text { TM incidence } \\ {\left[2 \frac{J_{1}\left(\left(k_{n}+k_{\mathrm{t}}\right) w / 2\right)}{\left(k_{n}+k_{\mathrm{t}}\right) w / 2}\right]^{2},} & \text { TE incidence. }\end{cases}
$$

Regarding the criterion for the number of low-order harmonics, we now define $N$ as the number of propagative negative harmonics $(n<0)$ plus 1 (the first evanescent one) inside the highest-permittivity medium at the highest frequency of interest, namely,

$$
N=\left\lceil\left(\sqrt{\varepsilon_{\mathrm{r}, \max }}+\sqrt{\varepsilon_{\mathrm{r}}^{(0)}} \sin \theta\right) \frac{p}{\lambda_{0, \min }}\right\rceil
$$

(for positive angles of incidence the number of propagative positive harmonics is at most equal to the number of propagative negative harmonics). All the harmonics with $|n| \leqslant N$ are thus considered low-order harmonics whose contribution to the above sums is taken rigorously without any approximation. The harmonics with $|n| \geqslant N+1$ are all far below the cutoff in the frequency range of interest, and thus we can use the following approximation:

$$
\begin{gathered}
k_{n}+k_{t} \approx k_{n}, \\
\beta_{n} \approx-\mathrm{j} k_{|n|},
\end{gathered}
$$

which implies that, once again, their global contribution to the equivalent circuit can be accounted for by frequencyindependent capacitances or inductances. Taking into account (55), the same criterion for $M$ in (28) can be applied here, and only those high-order harmonics with $|n| \leqslant M$ are considered in the elements that represent coupling through evanescent fields. 


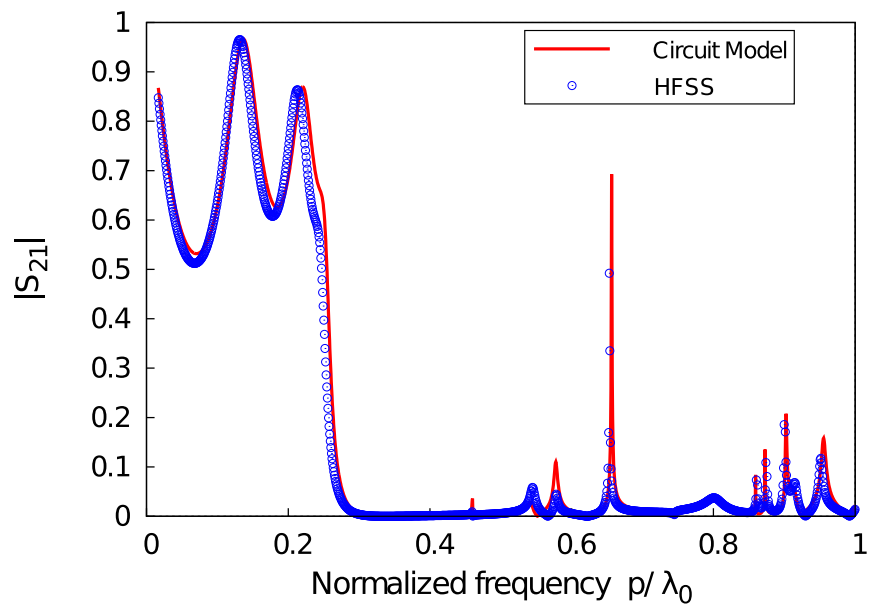

FIG. 15. Transmission coefficient (magnitude) versus normalized frequency for a four-slit-array stacked structure under oblique TM incidence $\left(\theta=20^{\circ}\right)$. Parameters: $w=0.1 p, d_{1}=0.4 p, d_{2}=0.3 p$, $d_{3}=0.2 p, \varepsilon_{\mathrm{r}}^{(0)}=1, \varepsilon_{\mathrm{r}}^{(1)}=2.2, \varepsilon_{\mathrm{r}}^{(2)}=4, \varepsilon_{\mathrm{r}}^{(3)}=3$.

As an example of application, Fig. 15 shows the reflection coefficient versus frequency for the case of four stacked slit arrays under oblique TM incidence with $\theta=20^{\circ}$. As in previous comparisons, the agreement with the results from HFSS is very good.

Finally, it should be mentioned that the range of application of the model is more reduced for oblique incidence than for normal incidence, as expected. As reasonable estimates of the validity limits, we have found that the model is valid for frequencies up to $w / \lambda_{\text {diel }} \lesssim 0.2$ for oblique TM incidence and $w / \lambda_{\text {eff }} \lesssim 0.5$ for oblique TE incidence.

\section{CONCLUSIONS}

A wideband equivalent $\Pi$ circuit has been derived for a pair of coupled slit arrays illuminated by TM or TE normally incident plane waves. Fully analytical expressions are derived for the $\Pi$-circuit elements, which are valid both for slightly and for tightly coupled gratings. The numerical series defining the circuit components are physically interpreted in terms of distributed (low-order-mode) and lumped (high-order-mode) contributions, in such a way that explicit networks can be obtained for each specific configuration and maximum operation frequency. The deduced $\Pi$-circuit model shows separate contributions of the internal and external fields to the parallel elements, which allows for a straightforward extension of the circuit model to the case of stacked structures with an arbitrary number of slit gratings separated by dielectric slabs. Specifically, the equivalent circuit is obtained just by cascading the elementary internal $\Pi$ building blocks. The model can also be used for the dispersion analysis of the Bloch modes of infinitely long periodic stacks. The extension of the formulation to oblique incidence is straightforward. The validity of the derived circuit models has been systematically verified by proper comparison with numerical data generated by the MoM, by the commercial simulator HFSS, and also by some available experimental results.

\section{ACKNOWLEDGMENTS}

This work was supported by the Spanish Ministerio de Economía y Competitividad with European Union FEDER funds (project TEC2013-41913-P) and by the Consejería de Economía, Innovación, Ciencia y Empleo, Junta de Andalucía (project P12-TIC-1435).

\section{APPENDIX: TRANSMISSION LINE EQUIVALENT TO A ח CIRCUIT}

The role of the low-order modes associated with the internal $\Pi$ topology in Sec. II B can be treated as individual $n$-order $\Pi$ circuits. The $n$-order modal parallel and series admittances are given by

$$
\begin{gathered}
Y_{\mathrm{p}, \mathrm{n}}^{(1)}=\mathrm{j} A_{n} Y_{n}^{(1)} \tan \left(\beta_{n}^{(i)} d_{1} / 2\right), \\
Y_{\mathrm{s}, \mathrm{n}}=-\mathrm{j} A_{n} Y_{n}^{(1)} \csc \left(\beta_{n}^{(1)} d_{1}\right) .
\end{gathered}
$$

Using the transmission matrix formulation [62], the $A B C D$ parameters associated with a $\Pi$ topology are given by

$$
[A B C D]_{n}=\left[\begin{array}{cc}
1+\frac{Y_{\mathrm{p}, n}^{(1)}}{Y_{\mathrm{s}, n}} & \frac{1}{Y_{\mathrm{s}, n}} \\
2 Y_{\mathrm{p}, n}^{(1)}+\frac{\left(Y_{\mathrm{p}, n}^{(1)}\right)^{2}}{Y_{\mathrm{s}, n}} & 1+\frac{Y_{\mathrm{p}, n}^{(1)}}{Y_{\mathrm{s}, n}}
\end{array}\right] .
$$

Substituting (A1) and (A2) into (A3), it is found after some manipulations that

$$
[A B C D]_{n}=\left[\begin{array}{ll}
Y_{n}^{(1)} \cos \left(\beta_{n}^{(1)} d_{1}\right) & \mathrm{j} \frac{A_{n}}{Y_{n}^{(1)}} \sin \left(\beta_{n}^{(1)} d_{1}\right) \\
\mathrm{j} \frac{Y_{n}^{(1)}}{A_{n}} \sin \left(\beta_{n}^{(1)} d_{1}\right) & Y_{n}^{(1)} \cos \left(\beta_{n}^{(1)} d_{1}\right)
\end{array}\right],
$$

which can be decomposed as the following matrix product:

$$
\begin{aligned}
{[A B C D]_{n}=} & {\left[\begin{array}{cc}
A_{n}^{1 / 2} & 0 \\
0 & \frac{1}{A_{n}^{1 / 2}}
\end{array}\right] } \\
& \times\left[\begin{array}{cc}
Y_{n}^{(1)} \cos \left(\beta_{n}^{(1)} d_{1}\right) & \mathrm{j} \frac{1}{Y_{n}^{(1)}} \sin \left(\beta_{n}^{(1)} d_{1}\right) \\
\mathrm{j} Y_{n}^{(1)} \sin \left(\beta_{n}^{(1)} d_{1}\right) & Y_{n}^{(1)} \cos \left(\beta_{n}^{(1)} d_{1}\right)
\end{array}\right] \\
& \times\left[\begin{array}{cc}
\frac{1}{A_{n}^{1 / 2}} & 0 \\
0 & A_{n}^{1 / 2}
\end{array}\right] .
\end{aligned}
$$

This cascade of transmission matrices actually represents a transmission-line section of length $d_{1}$ and characteristic admittance $Y_{n}^{(1)}$ terminated by transformers of the turn ratio $A_{n}^{1 / 2}$ on both sides [62]. Note that for $n=0, A_{0}=1$ for either $\mathrm{TE}$ or TM polarization, and thus (A5) reduces to

$$
\left[\begin{array}{cc}
Y_{0}^{(1)} \cos \left(\beta_{0}^{(1)} d_{1}\right) & \mathrm{j} \frac{1}{Y_{0}^{(1)}} \sin \left(\beta_{0}^{(1)} d_{1}\right) \\
\mathrm{j} Y_{0}^{(1)} \sin \left(\beta_{0}^{(1)} d_{1}\right) & Y_{0}^{(1)} \cos \left(\beta_{0}^{(1)} d_{1}\right)
\end{array}\right],
$$

which represents a transmission-line section of length $d_{1}$ and characteristic admittance $Y_{0}^{(1)}$. 
[1] J. Fraunhofer, Ann. D. Phys. 74, 337 (1823).

[2] R. W. Wood, Proc. Phys. Soc. London 18, 269 (1902).

[3] L. Rayleigh, Philos. Mag. 14, 60 (1907).

[4] L. Rayleigh, Proc. R. Soc. London 79, 399 (1907).

[5] R. W. Wood, Phys. Rev. 48, 928 (1935).

[6] G. G. Macfarlane, J. Inst. Electr. Eng. Part 3A 93, 703 (1946).

[7] J. Brown, Proc. IEE Part III: Radio Commun. Eng. 97, 45 (1950).

[8] J. Brown, Proc. IEE Part IV 100, 51 (1953).

[9] J. Brown and W. Jackson, Proc. IEE Part B: Radio Electr. Eng. 102, 11 (1955).

[10] Y. Yang, I. Kravchenko, D. Briggs, and J. Valentine, Nat. Commun. 5, 5753 (2014).

[11] A. Coves, B. Gimeno, J. Gil, M. V. Andrs, A. A. San-Blas, and V. E. Boria, IEEE Trans. Antennas Propag. 52, 2091 (2004).

[12] B. A. Munk, Frequency Selective Surfaces: Theory and Design (John Wiley and Sons, New York, 2000), p. 410.

[13] M. Al-Joumayly and N. Behdad, IEEE Trans. Antennas Propag. 57, 452 (2009).

[14] B. Gimeno, J. L. Cruz, E. A. Navarro, and V. Such, IEEE Trans. Antennas Propag. 42, 912 (1994).

[15] J. S. Cetnar, J. R. Middendorf, and E. R. Brown, Appl. Phys. Lett. 100, 231912 (2012).

[16] M. A. Hiranandani, A. B. Yakovlev, and A. A. Kishk, Proc. Int. Electr. Eng. Microw., Antennas, Propag. 153, 487 (2006).

[17] O. Luukkonen, C. Simovski, G. Granet, G. Goussetis, D. Lioubtchenko, A. V. Raisanen, and S. A. Tretyakov, IEEE Trans. Antennas Propag. 56, 1624 (2008).

[18] T. W. Ebbesen, H. J. Lezec, H. F. Ghaemi, T. Thio, and P. A Wolff, Nature (London) 391, 667 (1998).

[19] H. F. Ghaemi, T. Thio, D. E. Grupp, T. W. Ebbesen, and H. J. Lezec, Phys. Rev. B 58, 6779 (1998).

[20] M. Beruete, M. Sorolla, I. Campillo, J. S. Dolado, L. MartínMoreno, J. Bravo-Abad, and F. J. García-Vidal, Opt. Lett. 29, 2500 (2004).

[21] M. Beruete, M. Sorolla, I. Campillo, J. S. Dolado, L. MartínMoreno, J. Bravo-Abad, and F. J. García-Vidal, IEEE Trans. Antennas Propag. 53, 1897 (2005).

[22] N. Marcuvitz, Waveguide Handbook (McGraw-Hill, New York, 1951).

[23] R. E. Collin, Field Theory of Guided Waves (IEEE Press, Piscataway, NJ, 1991).

[24] A. G. Schuchinsky, D. E. Zelenchuk, A. M. Lerer, and R. Dickie, IEEE Trans. Antennas Propag. 54, 490 (2006).

[25] M. Beruete, I. Campillo, M. Navarro-Cía, F. Falcone, and M. Sorolla, IEEE Trans. Antennas Propag. 55, 1514 (2007).

[26] R. Gordon, Phys. Rev. A 76, 053806 (2007).

[27] A. A. Kirilenko and A. O. Perov, IEEE Trans. Antennas Propag. 56, 3210 (2008).

[28] Y. Pang, A. N. Hone, P. P. M. So, and R. Gordon, Opt. Express 17, 4433 (2009).

[29] F. Medina, F. Mesa, and R. Marqués, IEEE Trans. Microwave Theory Tech. 56, 3108 (2008).

[30] F. Medina, F. Mesa, and D. C. Skigin, IEEE Trans. Microwave Theory Tech. 58, 105 (2010).

[31] L. B. Felsen and A. A. Oliner, Proc. Inst. Radio Eng. 42, 477 (1954).

[32] M. Ohira, H. Deguchi, M. Tsuji, and H. Shigesawa, Electron. Commun. Japan 88, 9 (2005).
[33] F. Costa, A. Monorchio, and G. Manara, IEEE Antennas Propag. Mag. 54, 35 (2012).

[34] Y. Gong, X. Liu, K. Li, J. Huang, J. J. Martínez, D. Rees-Whippey, S. Carver, L. Wang, W. Zhang, T. Duan, and N. Copner, Phys. Rev. B 87, 205121 (2013).

[35] R. Rodríguez-Berral, F. Medina, and F. Mesa, Appl. Phys. Lett. 96, 161104 (2010).

[36] M. García-Vigueras, F. Mesa, F. Medina, R. Rodríguez-Berral, and J. L. Gómez-Tornero, IEEE Trans. Antennas Propag. 60, 4637 (2012).

[37] R. Rodríguez-Berral, F. Medina, F. Mesa, and M. GarcíaVigueras, IEEE Trans. Microwave Theory Tech. 60, 405 (2012).

[38] E. Yarmoghaddam, G. K. Shirmanesh, A. Khavasi, and K. Mehrany, IEEE Trans. Antennas Propag. 62, 4041 (2014).

[39] B. Blázquez, N. Llombart, D. Cavallo, A. Freni, and A. Neto, IEEE Trans. Antennas Propag. 62, 5077 (2014).

[40] R. Rodríguez-Berral, C. Molero, F. Medina, and F. Mesa, IEEE Trans. Microwave Theory Tech. 60, 3908 (2012).

[41] C. Molero, R. Rodriguez-Berral, F. Mesa, and F. Medina, IEEE Trans. Antennas Propag. 62, 794 (2014).

[42] F. Mesa, M. García-Vigueras, F. Medina, R. Rodríguez-Berral, and J. Mosig, IEEE Antennas Wireless Propag. Lett. 14, 135 (2015).

[43] S. Monni, G. Gerini, and A. Neto, IEEE Trans. Antennas Propag. 55, 2824 (2007).

[44] M. Beruete, M. Navarro-Cía, and M. Sorolla, IEEE Trans. Microwave Theory Tech. 59, 2180 (2011).

[45] L. A. Vainshtein, Zh. Tekh. Fiz. 25, 847 (1955); translated in Sov. Phys. Tech. Phys.

[46] Z. S. Agranovich, V. A. Marchenko, and V. P. Shestopalov, Engl. Transl. Sov. Phys. Tech. Phys. 7, 277 (1962).

[47] V. G. Yampolsky, Radiotek. Elektron. 8, 564 (1963); translated in Radio Eng. Electron. Phys.

[48] L. N. Litvinenko, Zh. Vychislitel. Mat. Mat. Fiz. 10, 99 (1970); translated in Sov. J. Comput. Math. Math Phys.

[49] K. Kobayashi, Can. J. Phys. 63, 453 (1985).

[50] Y. V. Pimenov and M. Y. Chervenko, Radiotek. Elektron. 31, 1088 (1986); translated in J. Commun. Technol. Electron.

[51] K. Kobayashi, IEEE Trans. Antennas Propag. 37, 459 (1989).

[52] T. L. Zinenko, A. I. Nosich, and Y. Okuno, IEEE Trans. Antennas Propag. 46, 1498 (1998).

[53] T. L. Zinenko and A. I. Nosich, IEEE Trans. Antennas Propag. 54, 2088 (1998).

[54] A. P. Hibbins, J. R. Sambles, C. R. Lawrence, and J. R. Brown, Phys. Rev. Lett. 92, 143904 (2004).

[55] R. Marqués, L. Jelinek, F. Mesa, and F. Medina, Opt. Exp. 17, 11582 (2009).

[56] C. S. R. Kaipa, A. B. Yakovlev, F. Medina, F. Mesa, C. A. M. Butler, and A. P. Hibbins, Opt. Express 18, 13309 (2010).

[57] C. Molero, R. Rodríguez-Berral, F. Mesa, F. Medina, and A. B. Yakovlev, in Proceedings of 2014 International Microwave Symposium (IEEE, New York, 2014).

[58] V. Delgado, R. Marqués, and L. Jelinek, IEEE Trans. Antennas Propag. 61, 1342 (2013).

[59] I. M. Mandel, A. B. Golovin, and D. T. Crouse, Phys. Rev. A 87, 053847 (2013). 
[60] D. Cavallo, W. H. Syed, and A. Neto, IEEE Trans. Antennas Propag. 62, 6256 (2014).

[61] D. Cavallo, W. H. Syed, and A. Neto, IEEE Trans. Antennas Propag. 62, 6265 (2014).

[62] D. M. Pozar, Microwave Engineering (John Wiley \& Sons, New York, 2005).

[63] V. Torres, F. Mesa, M. Navarro-Cía, R. Rodríguez-Berral, M. Beruete, and F. Medina, IEEE Trans. Microwave Theory Technol. 64, 15 (2016).
[64] V. Torres, P. Rodríguez-Uribarri, M. Navarro-Cía, and M. Beruete, Appl. Phys. Lett. 101, 244101 (2012).

[65] F. Medina and M. Horno, IEEE Trans. Microwave Theory Technol. 40, 1748 (1992).

[66] G. Cano, F. Medina, and M. Horno, IEEE Trans. Microwave Theory Technol. 46, 1801 (1998).

[67] ANSYS, High frequency structure simulator; http://www.ansys. com/Products/. Accessed: 27 December 2015. 